INPLASY

PROTOCOL

To cite: Yu et al. A scoping review protocol of oral Chinese traditional medicine for vascular dementia. Inplasy protocol 202170057. doi: 10.37766/inplasy2021.7.0057

Received: 19 July 2021

Published: 19 July 2021

Corresponding author: Jun Tang

wsyzmyxy@163.com

Author Affiliation:

Chongqing Hospital of

Traditional Chinese Medicine.

Support: Project BEBPC-TCM.

Review Stage at time of this submission: Preliminary searches.

Conflicts of interest:

None declared.

\section{A scoping review protocol of oral Chinese traditional medicine for vascular dementia}

Yu, X1; Guan, Y2; Yang, J3; Xiong, X4; Li, X5; Shu, J6; Qu, X7; Gao, X8; Tao, W9; Mi, X10; Luo, X11; Lian, Z12; Zhao, Y13; Liao, $X^{14}$.

Background: Vascular dementia is a common form of dementia which is caused by problems with the blood supply to the brain. Some but not all people with vascular dementia have had strokes. Western medicines licensed to treat vascular dementia are limited. Oral Chinese traditional medicines are often used to treat it in China. Oral Chinese traditional medicine in this review is defined as the oral Chinese Patent Medicine with a certain dosage form of medicinal substances recorded in Chinese materia medica or used in folk medicine. It is a commercial Chinese medicine preparation with standardized in source, nature, collection, processing, dispensing, actions, effects and uses, and approved by the National Health Commission of the People's Republic of China (NHC) and National Medical Products Administration of China (NMPA). Oral Chinese traditional medicines are categorized according to the nature of medicinals (the basic properties of a medicinal). Despite of symptomatic descriptions as 'dementia', 'memory loss', and 'Cognitive deficit' in some of the drug instructions, drug instructions rarely include 'vascular dementia' as one of the indications specifically. However, increasing studies on oral Chinese traditional medicines for the treatment of $\mathrm{VaD}$ give thoughts to deliberate about their applications for VaD. Recent studies of oral Chinese traditional medicine have demonstrated in vitro biological activity and therapeutic effects in animals, but the published clinical evidence has not been systematically appraised.

INPLASY registration number: This protocol was registered with the International Platform of Registered Systematic Review and Meta-Analysis Protocols (INPLASY) on 19 July 2021 and was last updated on 19 July 2021 (registration number INPLASY202170057).

\section{INTRODUCTION}

Review question / Objective: To conduct a systematic comprehensive review for 17 oral Chinese traditional medicines which are universally applied for use in vascular dementia and categorized in Chinese National Essential Health Insurance 
Program (2020 edition, CNEHIP), Chinese National Essential Medicines List (2018 edition, CNEML), or Chinese Pharmacopoeia (2020 edition, CP), and to identify the types of available evidence in this field as a precursor to a systematic review.

Rationale: Oral Chinese traditional medicines are widely used for $\mathrm{VaD}$ in China, while that is increasingly demonstrated by various researches. However, with the heterogeneity between the interventions and qualities of the current studies, it is necessary to provide a comprehensive overview of current clinical evidence. Scoping review is the process of determining the scope, type, and nature of evidence in a research area, systematically reviewing the value of the evidence, drawing conclusions from methodologically or disciplinarily distinct bodies of knowledge, or identifying research gaps to aid in planning and future research directions. Therefore, we conducted this scoping review on oral Chinese traditional medicine for vascular dementia to overview the current evidence and reflect the current research gaps by based on the PRISMAScR Checklist, including all guidelines, consensuses, meta-analyses, system reviews, and randomized controlled trials on 17 oral Chinese traditional medicines for vascular dementia.

Background: Vascular dementia is a common form of dementia which is caused by problems with the blood supply to the brain. Some but not all people with vascular dementia have had strokes. Western medicines licensed to treat vascular dementia are limited. Oral Chinese traditional medicines are often used to treat it in China. Oral Chinese traditional medicine in this review is defined as the oral Chinese Patent Medicine with a certain dosage form of medicinal substances recorded in Chinese materia medica or used in folk medicine. It is a commercial Chinese medicine preparation with standardized in source, nature, collection, processing, dispensing, actions, effects and uses, and approved by the National Health Commission of the People's
Republic of China (NHC) and National Medical Products Administration of China (NMPA). Oral Chinese traditional medicines are categorized according to the nature of medicinals (the basic properties of a medicinal). Despite of symptomatic descriptions as 'dementia', 'memory loss', and 'Cognitive deficit' in some of the drug instructions, drug instructions rarely include 'vascular dementia' as one of the indications specifically. However, increasing studies on oral Chinese traditional medicines for the treatment of VaD give thoughts to deliberate about their applications for VaD. Recent studies of oral Chinese traditional medicine have demonstrated in vitro biological activity and therapeutic effects in animals, but the published clinical evidence has not been systematically appraised.

\section{METHODS}

Search strategy: The oral Chinese traditional medicines included were as follows: BuShenYiNao, ShenWuJianNao (KangNaoShuai [former name]), CongRongYiShen, DengZhanShengMai, FuFangCongRongYiZhi (CongSheng Capsule[former name]), FuFangDanShen, GuilingJi, JianNa o, Na oMaiTai, NaoXinTong, TianMaXingNao, TianZhi, TongXinLuo, XinNaoJian, YiXinNingShen, YinDanXingNaoTong, and Ginkgo Biloba Leaf. We searched PubMed, EMBASE, Cochrane, and four major Chinese electronic databases, China National Knowledge Infrastructure (CNKI), China Science and Technology Journal Database (VIP), China BioMedical Literature Service System (SinoMed), and Wanfang Database. Seventeen Oral Chinese traditional medicines were searched independently. The search syntax was composed of 'vascular dementia', 'guidelines', 'consensuses', 'meta-analyses', 'system reviews', 'randomized controlled trials', English and Chinese Drug names and their synonyms. The search imposed no limitation on language. The basic information on the 17 oral Chinese traditional medicine was summarized according to the drug indications, Chinese Patent Medicinal Prescriptions Database of 
DRUGDATAEXPY, CNEHIP, CNEML, and CP. The quantitative description and figures were conducted with Microsoft Excel 2019, GraphPad prism software (GraphPad; San Diego, California, United States) and RStudio statistical software (RStudio; Boston, Massachusetts, United States). This scoping review was conducted and reported according to the Preferred Reporting Items for Systematic Reviews and Meta-Analyses Extension for Scoping Reviews (PRISMA-SCRs) checklist.

Eligibility criteria: All guidelines, consensuses, meta-analyses, system reviews, and randomized controlled trials (RCTs) on oral Chinese traditional medicines for vascular dementia were included. The oral Chinese traditional medicines included were as described a bove, namely: BuShenYiNao, ShenWuJianNao (KangNaoShuai [former name ]), CongRongYiShen, D e $n g Z h$ a $n S$ e $n g M a i$, FuFangCongRongYiZhi (CongSheng Capsule[former name]), FuFangDanShen, GuilingJi, JianNao, NaoMaiTai, NaoXinTong, TianMaXingNao, TianZhi, TongXinLuo, XinNaoJian, YiXinNingShen, YinDanXingNaoTong, and Ginkgo Biloba Leaf. We defined a study as a RCT if the allocation was described as randomized (including terms such as 'randomly', 'random' or 'randomization'). If no further description of the method was provided, we still included the study but appraised it as no information (NI) in allocation sequence randomization and allocation sequence concealing (signaling questions 1.1 and 1.2) in Cochrane risk-of-bias tool for randomized trials (Rob 2.0). No limitations were imposed on language, year, and publication status. Conference abstracts without details were excluded. The included guidelines, consensuses, meta-analyses, and system reviews should: (1) focus on the medicine in search strategy (defined as the only medicine in title and key words); (2) contain a description of clinical application of the medicine in VaD. For RCTs, participants had to be diagnosed with $\mathrm{VaD}$ by any of the following four criteria: (1) Diagnostic and Statistical Manual of Mental Disorders (DSM) versions
III, III-R, IV, IV-TR; (2) National Institute of Neurological Disorders and Stroke (NINDSAIREN); (3) International Classification of Diseases 9 or 10; (4) the Hachinski or the Modified Hachinski Ischaemic Score. We required the use of an imaging technique to differentiate VaD from other dementias. We excluded trials with participants diagnosed with mixed dementia or those that did not use an imaging technique to ascertain VaD. There were no restrictions on age and gender. Types of interventions were defined as those with at least one experimental intervention includes the medicine in search strategy. Other interventions, such as conventional pharmacological interventions, placebo and routine therapy (RT) for control of cerebrovascular risk factors, were accepted as combined therapies or control interventions. RT would typically be pharmacologic agents such as antihypertensives, anti-diabetics, antiplatelets, lipid-lowering drugs and blood flow-improving drugs. We also accepted nonpharmacologic agents as RT when they were clearly described by the authors as being used for such a purpose. Studies were excluded when all the interventions include the medicine in search strategy. There were no limitations on dosage, duration, and outcome.

Source of evidence screening and selection: The authors (XYY and YYG) reviewed the titles and abstracts of all retrieved references after removing duplicates, and the full-text was retrieved for further screening. XJM and XQL checked all the eligible studies and any disagreements were solved by discussion with other authors. The inclusion of trials was confirmed upon consensus of reviewers before data extraction. Data extraction was conducted with structured tables by XYY, YYG, XX, CYL, JZS, XLQ, $X F G, W Q T, X J M, Z Y L$, and $Y H Z$ independently, and validated by JT, JY and $X L$. All investigators were briefed and trained on using the data extraction tables beforehand to ensure accurate and consistent data extraction and assessment. Discrepancies were resolved by examining the original papers and by discussion. 
Data management: Three different data extraction tables (supplementary material: S2 Appendix) were specifically designed for (1) guidelines and consensuses, (2) metaanalyses and SRs, (3) RCTs to capture the required data from different types of study. In general, the extracted data of different types of study include the following: (i) Guidelines and consensuses: title, authors, publication year, funding, and medical effects on VaD. (ii) Meta-analyses, SRs: title, authors, publication year, funding, sample size, population type, Interventions, and controls. (iii) RCTs: study characters (title, authors, publication year, funding, single/multiple centers, hospital level for trials conducted in China [1, 2a, 2b, 3a, 3b], diagnostic criteria, randomization methods, blinding), participants' characters (sample size, TCM syndrome classification), intervention and comparator details (formulation, dose, frequency, duration, combined intervention, cointervention), outcome measures, and adverse events. Bias risk assessment of intention-to-treat (ITT) effect was conducted by XYY and YYG with The Revised Cochrane risk-of-bias tool (RoB 2) provided in the Cochrane Handbook for Systematic Reviews of Interventions.

Language: None restriction.

Country(ies) involved: China.

Keywords: oral Chinese traditional medicine, vascular dementia, scoping review, protocol.

Contributions of each author:

Author 1 - Xinyuan Yu.

Author 2 - Yueyue Guan.

Author 3 - Jian Yang.

Author 4 - Xin Xiong.

Author 5 - Chenyu Li.

Author 6 - Jianzhong Shu.

Author 7 - Xiaoli Qu.

Author 8 - Xiaofei Gao.

Author 9 - Wenqiang Tao.

Author 10 - Xiujuan Mi.

Author 11 - Xiaoqiong Luo.

Author 12 - Zhiyun Lian.

Author 13 - Yuhua Zhao.

Author 14 - Xing Liao. 